MATEC Web of Conferences 32, 03002 (2015)

DOI: $10.1051 /$ matecconf $/ 20153203002$

C) Owned by the authors, published by EDP Sciences, 2015

\title{
3D micro-optical elements for generation of tightly focused vortex beams
}

\author{
Armandas Balčytis ${ }^{1,2, a}$, Davit Hakobyan ${ }^{1,3}$, Martynas Gabalis², Albertas Žukauskas ${ }^{4}$, Darius Urbonas², \\ Mangirdas Malinauskas ${ }^{4}$, Raimondas Petruškevičius ${ }^{2}$, Etienne Brasselet ${ }^{3}$, and Saulius Juodkazis ${ }^{1,5}$ \\ ${ }^{1}$ Centre for Micro-Photonics, Faculty of Engineering and Industrial Sciences, Swinburne University of Technology, Hawthorn, VIC \\ 3122, Australia \\ ${ }^{2}$ Institute of Physics, Center for Physical Sciences and Technology, 231 Savanoriu Avenue, LT-02300 Vilnius, Lithuania \\ ${ }^{3}$ University of Bordeaux, CNRS, Laboratoire Ondes et Matière d'Aquitaine, 351 cours de la libération, F-33400 Talence, France \\ ${ }^{4}$ Department of Quantum Electronics, Physics Faculty, Vilnius University, Saulètekio Ave. 10, LT-10223 Vilnius, Lithuania \\ ${ }^{5}$ Melbourne Centre for Nanofabrication, 151 Wellington Road, Clayton, VIC 3168, Australia
}

\begin{abstract}
Orbital angular momentum carrying light beams are usedfor optical trapping and manipulation. This emerging trend provides new challenges involving device miniaturization for improved performance and enhanced functionality at the microscale. Here we discus a new fabrication method based on combining the additive 3D structuring capability laser photopolymerization and the substractive sub-wavelength resolution patterning of focused ion beam lithography to produce micro-optical elements capable of compound functionality. As a case in point of this approach binary spiral zone pattern based high numerical aperture micro-lenses capable of generating topological charge carrying tightly focused vortex beams in a single wavefront transformation step are presented. The devices were modelled using finite-difference time-domain simulations, and the theoretical predictions were verified by optically characterizing the propagation properties of light transmitted through the fabricated structures. The resulting devices had focal lengths close to the predicted values of $f=18 \mu \mathrm{m}$ and $f=13 \mu \mathrm{m}$ as well as topological charge $\ell$ dependent vortex focal spot sizes of $\sim 1.3 \mu \mathrm{m}$ and $\sim 2.0 \mu \mathrm{m}$ for $\ell=1$ and $\ell=2$ respectively.
\end{abstract}

\section{Introduction}

Orbital angular momentum (OAM) of an optical vortex presents a new dimension for controlling light-matter interaction, which confers substantial benefits to a multitude of application areas. Optical tweezers/spanners that use vortices to trap particles can do so with higher axial trapping efficiency compared to a Gaussian beam of equivalent intensity, which is of special importance in biological applications where reduction in the laser power used diminishes the risk of sample damage [1]. Furthermore, they provide additional functionality by enabling orbital rotation on top of typical spinning motion as well as stable trapping of absorbing particles [2]. Similarly, optical vortex phase masking is a technique of significant interest in astronomy, where it can be used to distinguish faint objects close to bright coherent sources for producing coronagraphs and searching of exoplanets [3, 4]. Optical communications is another field where the theoretically unlimited range of topological charge $\ell$ (where $\ell$ indicates the winding number of helical wavefront) in OAM carrying beams shows great promise [5].

The physical phenomenon underlying the aforementioned functions is the Laguerre-Gaussian (LG) beam or Bessel beam, which exhibits annular transverse intensity profiles and possesses an azimuthal phase dependence

\footnotetext{
a e-mail: abalcytis@swin.edu.au
}

given by $\exp (i \ell \varphi)$ carrying a well defined OAM of $\ell \hbar$ per photon, where $\hbar$ is the reduced Planck's constant and $\varphi$ is the azimuthal coordinate [6]. The most obvious feature of such a vortex beam is that the azimuthal angle, hence, the phase becomes indeterminate at its center. Thus, in order to retain consistency, the amplitude of light at the beam center must decrease to zero to prevent the formation of a physical singularity.

The conventional methods of generating LG beams of either integral of fractional topological charge involve the use of spatial light modulators, forked diffraction gratings, phase plates or mode converters [2,7]. However, recent efforts to achieve optical trapping and manipulation at the microscale resulted in forgoing these bulky setups in favor of miniaturized structures, such as spiral zone plates (SZP) of both diffractive [8] and plasmonic [9] varieties, topological nanoslits [10], photopolymerized structures [11] as well as metasurface-based devices [12]. Although optical manipulation setups realized on the microscale provide for added versatility and integration, at the same time new challenges arise, such as having to find ways to supplant traditional tabletop optical setups for focusing and other types of wavefront manipulation. One strategy for achieving this is integration of the required functions into a single microscopic optical element.

This is an Open Access article distributed under the terms of the Creative Commons Attribution License 4.0, which permits unrestricted use, distribution, and reproduction in any medium, provided the original work is properly cited. 
Here, we propose a strategy of combining photopolymerization, able of creating transparent 3D microstructures for phase front shaping, and focused ion beam (FIB) milling through a metal mask, well suited for patterning of binary diffractive elements, to create micro-optical structures with compound functionality. As a case example 3D SZP structures fashioned by ion beam lithography on a photopolymerized pedestal and capable of generating tightly focused vortex beams with topological charges $\ell=1,2$ in a single step are presented. The chosen spiral zone pattern is a convolution of the radial Hilbert transform, responsible for the azimuthal phase profile, and Fresnel zone plate pattern, giving rise to the focusing behaviour. Herein we outline the steps involved in creating such structures, starting by detailing preliminary numerical investigation by means of finite-difference timedomain (FDTD) simulations, describing of the fabrication procedure, as well as discussing the optical characterization results.

\section{Techniques and methods}

Numerical simulations of wavefront shaping by the 3D SZP structures were conducted using a commercially available FDTD software package (FDTD Solutions, Lumerical Solutions, Inc.). The spiral patterns were generated by calculating the binary transmittance function of a SZP [13]:

$$
t\left(r^{2}, \varphi\right)= \begin{cases}0 & (2 k-1) \pi \leq \ell \varphi-\frac{\pi r^{2}}{\lambda f} \leq 2 k \pi \\ 1 & 2 k \pi \leq \ell \varphi-\frac{\pi r^{2}}{\lambda f} \leq(2 k+1) \pi\end{cases}
$$

where the polar coordinates in the SZP plane are denoted as $(r, \varphi), \lambda$ is the wavelength of monochromatic light, $f$ is the corresponding focal length and index $k=0,-1,-2 \ldots$ defines the spiral zone number. The overall diameter of the SZP was chosen to be $R=30 \mu \mathrm{m}$ with a corresponding minimum $k=-10$. The spiral pattern was subsequently incorporated onto a geometry that closely approximates the subsequently manufactured gold coated photopolymerized structures on a glass substrate.

The simulated structures were excited from the substrate side using $\lambda=532 \mathrm{~nm}$ wavelength circularly polarized light of matching handedness, obtained by superimposing two orthogonal linearly polarized light sources with a $\pi / 2$ phase offset. The simulation region was terminated by perfectly matched layer boundaries.

Fabrication of the 3D SZP structures involved the photopolymerization of the disk or cone shaped pedestal, followed by coating it with an optically opaque layer of $\mathrm{Au}$, into which the spiral zone patterns were subsequently milled by means of ion beam lithography.

3D photopolymerization by direct laser writing was conducted using a hybrid organic-inorganic photopolymer SZ2080 doped with Irgacure 369 photoinitiator at 2 wt. \% [14]. This polymer is particularly suitable for fashioning various micro-optical structures, since it is known to possess the required mechanical robustness as well as a refractive index closely matched to that of glass in the visible spectral region $\left(n_{S Z 2080}=1.504, n_{\text {Glass }}=1.52\right)[15]$.

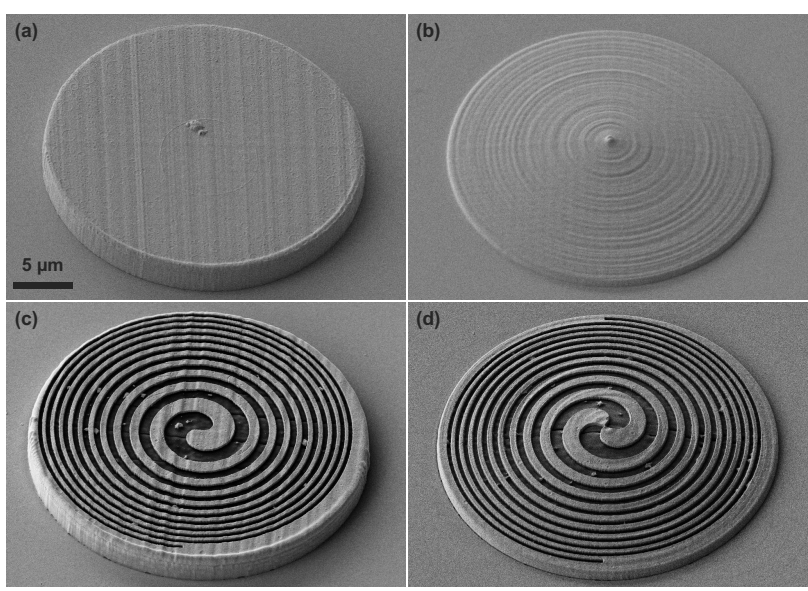

Figure 1. SEM images of photopolymerized flat disk pedestal (a) and obtuse axicon (b) structures after sputter-coating with 200 $\mathrm{nm}$ of Au. (c,d) SEM images of the same structures after FIB milling of SZP patterns with vortex orders $\ell=1$ (c) and $\ell=2$ (d) in the Au layer.

Prior to laser exposure the photoresist was prepared by drop-casting SZ2080 onto a cover-glass substrate, and was then prebaked on a hotplate for $60 \mathrm{~min}$ at $90{ }^{\circ} \mathrm{C}$. Direct-write fabrication was conducted using a $\mathrm{Yb}: \mathrm{KGW}$ (PHAROS, Light Conversion Ltd.) laser with 300 fs optical pulse duration at second harmonic $(\lambda=515 \mathrm{~nm}), 200$ $\mathrm{kHz}$ repetition rate, under tight focusing conditions using a $63 \times, N A=1.4$ microscope objective (Carl Zeiss). The $15 \mu \mathrm{m}$ radius $3 \mathrm{D}$ flat disk pedestal structures were produced under $70 \mu \mathrm{W}\left(0.988 \mathrm{TW} / \mathrm{cm}^{2}\right)$ laser intensity at focus, using a $0.5 \mathrm{~mm} / \mathrm{s}$ scanning velocity along raster trajectories with $50 \mathrm{~nm}$ voxel overlap. Conversely, the axicon structures with a full apex angle of $165^{\circ}$ were fabricated using $27.5 \mu \mathrm{W}\left(0.388 \mathrm{TW} / \mathrm{cm}^{2}\right)$ laser intensity at $0.1 \mathrm{~mm} / \mathrm{s}$ scanning velocity along spiral trajectories with $50 \mathrm{~nm}$ voxel overlap. Photopolymer development was performed by immersing it into pure 4-methyl-2-pentanone for $1 \mathrm{~h}$ to remove any unexposed residue.

The resulting photopolymerized structure was then coated with a $200 \mathrm{~nm}$ thick Au film using magnetron sputtering (AXXIS, JKLesker). Spiral zone patterns where transferred onto the Au sputtered polymerized structures by means of FIB milling, using a Raith IonLINE ion beam lithography tool. After precise alignment of the polymerized structure with the center of the $50 \mu \mathrm{m}$ writefield, a $35 \mathrm{kV} \mathrm{Ga} a^{+}$ion beam was focused down to a $25 \mathrm{~nm}$ diameter spot and set to scan along the spiral pattern until the $200 \mathrm{~nm}$ Au layer was completely removed. Representative SEM images of the structures before and after FIB milling are shown in Fig. $1(\mathrm{a}, \mathrm{b})$ and $(\mathrm{c}, \mathrm{d})$ respectively.

Optical characterization of the fabricated devices involved investigation of the propagation features of the transmitted light field. Excitation of the structures was performed at normal incidence from the glass substrate side using a $532 \mathrm{~nm}$ wavelength laser. The circularly polarized fundamental Gaussian probe beam was focused at the device plane into a spot which exceeds the area of the spiral 


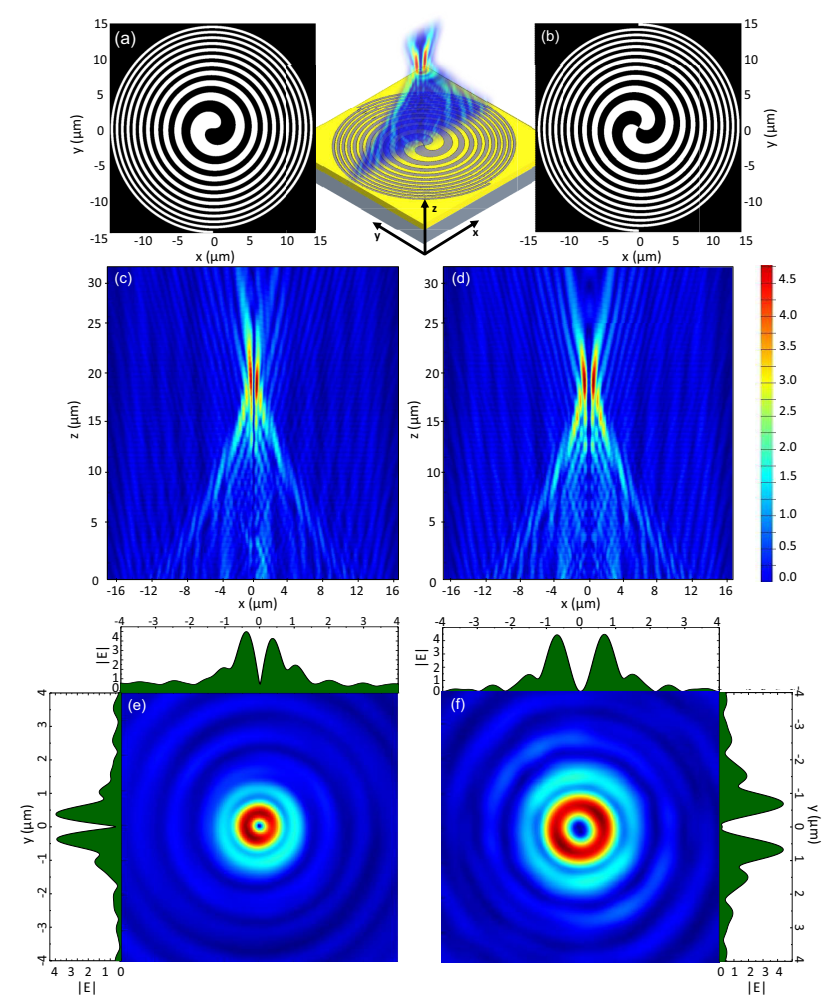

Figure 2. (a,b) SZP transmittance patterns for vortex orders $\ell=1$ (a) and $\ell=2$ (b) with projected focal distances of $18 \mu \mathrm{m}$ devised for $\lambda=532 \mathrm{~nm}$ wavelength. Cartoon inset in the middle illustrates the coordinate conventions used for the simulations. (c,d) FDTD simulated electric field intensity distributions of $\lambda=$ $532 \mathrm{~nm}$ light, diffracted through SZP with vortex orders $\ell=1$ (c) and $\ell=2(\mathrm{~d})$, obtained in the $x z$ plane parallel to the direction of propagation. (e,f) FDTD simulated electric field intensity distributions in the $x y$ plane at the focal distance of the SZP for vortex orders $\ell=1$ (e) and $\ell=2$ (f). Plots in green show corresponding lateral cross-sections through the intensity plot.

zone pattern, with the ratio of incident beam waist size $w_{0}$ and the radius of the structure $R$ chosen to be $w_{0} / R=2$. The optical intensity profiles at a distance $z$ from the plane of the structure were recorded by means of a microscope objective $(100 \times, N A=0.8)$ and a CCD camera. The corresponding phase profiles were determined by means of recording an interference pattern with a collinear Gaussian reference arm.

\section{Results and discussion}

Numerical FDTD simulations of the transmittance of right hand circularly polarized light through the flat spiral zone structures predict strongly focusing behaviour, with the focal distance being very close to the value of $f=18 \mu \mathrm{m}$ set by equation 1 used to generate the binary transmittance pattern. As shown for examples with vortex orders $\ell=1,2$ in Fig. 2, regardless of the topological charge of a chosen spiral zone pattern the focal distance, hence, the numerical aperture of vortex lenses remains constant, evaluated to be $N A \approx 0.64$ based on the marginal ray of the structure. When considering the simulated optical intensity profiles

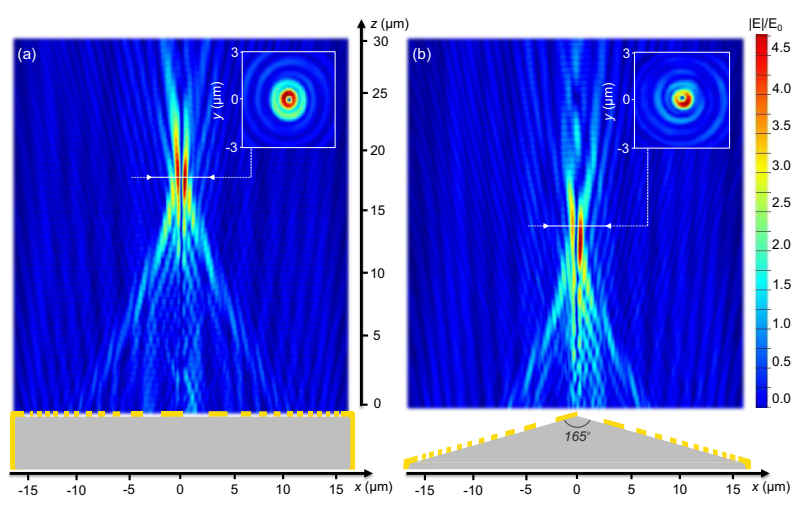

Figure 3. FDTD simulated electric field intensity distributions of $\lambda=532 \mathrm{~nm}$ light, diffracted through SZP with orders $\ell=1$ generated on $3 \mathrm{D}$ photopolymerized flat disk pedestal (a) and cone with an obtuse apex angle of $165^{\circ}(\mathrm{b})$, obtained in the $x z$ plane parallel to the direction of propagation. Insets show enhanced field intensity profiles in a $3 \times 3 \mu \mathrm{m}$ region in the $x z$ plane at the respective focal distances.

at the focal plane, presented in Fig. 2 (e) and (f), it is apparent that the annular intensity pattern with a dark hollow in the middle is characteristic of a LG beam. Furthermore, the increased radius of the doughnut shaped intensity pattern for the $\ell=2$ structure lends further credence that an optical vortex is formed.

The intensity patterns at focal plane are close to being diffraction-limited for the $\lambda=532 \mathrm{~nm}$ light used, with the intensity ring diameters being $\approx 1.4 \mu \mathrm{m}$ and $\approx 2.1 \mu \mathrm{m}$ at FWHM for the $\ell=1$ and $\ell=2$ structures respectively. Another disparity between the focused vortices generated by the different order spirals lies in the symmetry of the intensity distributions. As can be observed in the lateral intensity cross-section plots in Fig. 2 (e) and (f), the first order spiral structure, owing to its antisymmetric transmittance pattern, produces a somewhat less uniform intensity throughout the ring when compared to the second order spiral, which has inversion symmetry. Further simulations show that uniformity in the focal intensity pattern can be increased by extending the spiral to have more zones, hence, larger diameter. Therefore, there is a tradeoff between focal uniformity and structure size. Furthermore, it is important to note that the focal distance $f$ and the operational wavelength $\lambda$ appear as a product in equation 1, hence, in typical Fresnel lens fashion, use of polychromatic light would result in multiple foci appearing along the device axis with each focal distance being inversely proportional to the wavelength. Similarly, the spiral zone structure can be tailored to yield a certain focal distance for any given wavelength.

When the spiral zone pattern is generated on a flat $3 \mu \mathrm{m}$ high 3D disk structure (Fig. 2 (a)) the light propagation behaviour as calculated in FDTD does not markedly change form the flat substrate case. On the other hand, when an axicon with a full apex angle of $165^{\circ}$ is used as the pedestal, certain alterations in the simulated focusing behaviour of the structure are observed (Fig. 2 (b)). The most obvious change is the decrease in focal length form 

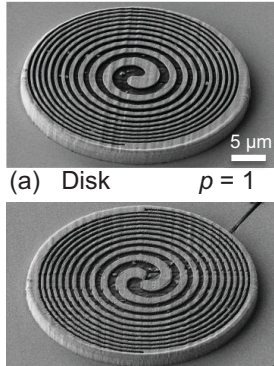

(b) Disk
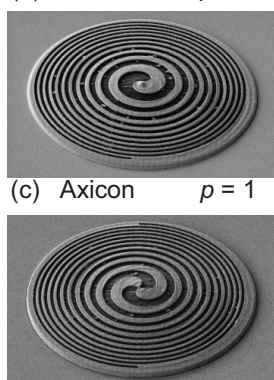

(d) Axicon
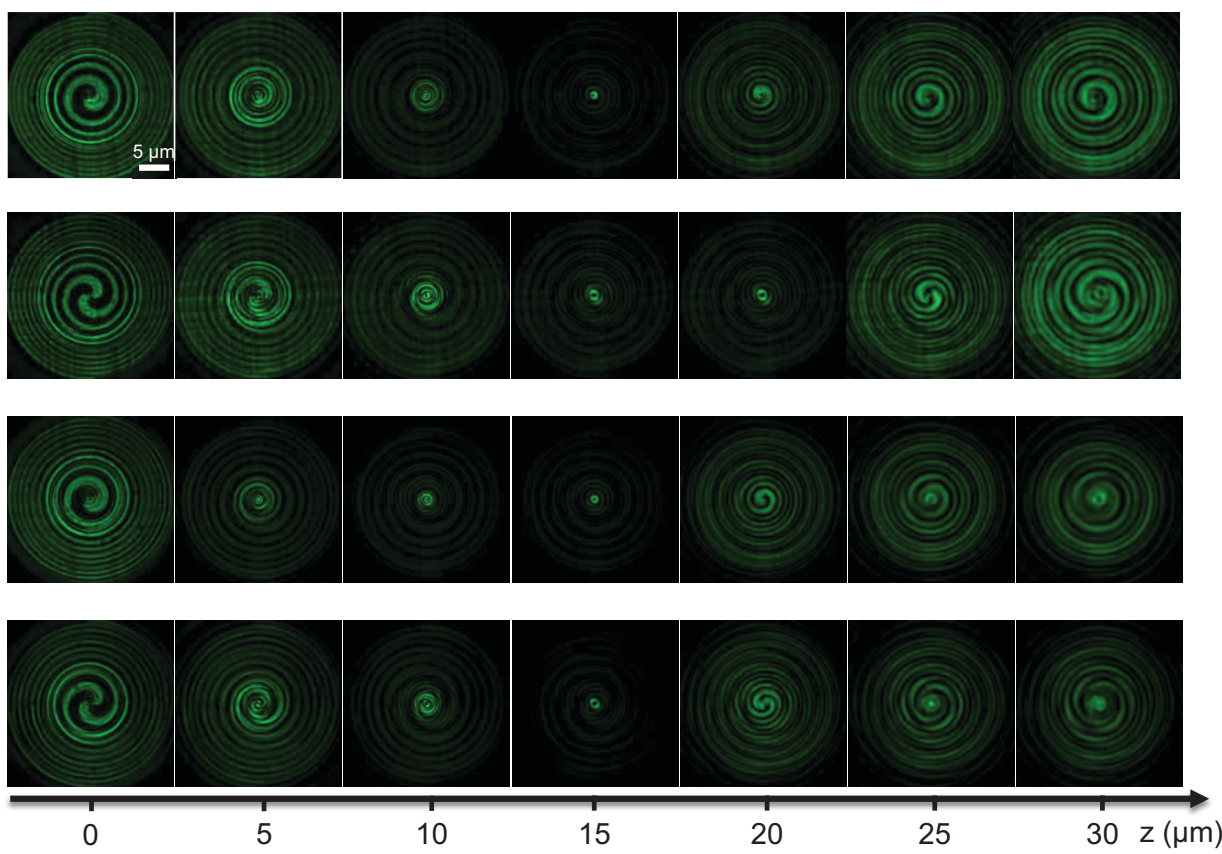

Figure 4. Propagation of the optical field upon transmittance through the SZP structures. Rows (a) and (b) correspond to SZP structures with vortex orders of $\ell=1$ and $\ell=2$ respectively, milled on a disk pedestal. Rows (c) and (d) show the optical fields transmitted through $\ell=1$ and $\ell=2$ vortex order structures respectively, milled on an axicon pedestal. First image in row refers to the structure plane $(\mathrm{z}=0)$. Propagation distance increases from left to right with step size $\Delta \mathrm{z}=5 \mu \mathrm{m}$. Imaging was done using a $100 \mathrm{x}$ NA $=0.8$ objective.

$f \approx 18 \mu \mathrm{m}$ down to $f \approx 13 \mu \mathrm{m}$, corresponding to a numerical aperture increase from $N A \approx 0.64$ to $N A \approx 0.76$. This is due to the phase front deformation introduced by the axicon, with phase delays for light propagating through spiral zones close to the apex of the cone being larger than at the edges of the structure. Furthermore, use of an axicon pedestal leads to a further decrease in the uniformity of intensity at the focal spot. However, a decrease in the diameter of the annular vortex intensity pattern is observed, which can be of significant benefit when stronger localization in high topological charge beams is required. Simulations of spiral zone patterns generated on a concave axicon pedestal (inverted case of Fig. 2 (b), results not shown) indicate that opposite behavior is observed, i.e. focal distance is increased, uniformity at focus is enhanced and vortex spot size is expanded.

In order to conduct experimental verification of the behavior predicted by FDTD simulations, the 3D spiral zone structures with orders $\ell=1,2$ were fabricated by means FIB lithography on both flat disk and obtuse axicon pedestals created using direct-write laser photopolymerization. Interferometrically controlled piezoelectric stage as well as FIB imaging capability allowed for precise control over the positioning of the pedestal structures for spiral pattern transfer. The $200 \mathrm{~nm}$ thick gold layer was selectively stripped over $10^{4}$ sequential patterning loops. The binary transmission patterns were thus reproduced with high fidelity as shown in Fig. 1, closely approximating the geometric parameters deduced from numerical simulations. Some residual gold islands can still be observed in the stripped sections, most likely resulting from impu- rities on the surface of the polymerized pedestal acting as crystallization sites for the sputtered Au, making it more resilient to ion bombardment then the rest of the structure.

Optical characterization was performed for the purpose of experimentally determining the transmittance behaviour of the fabricated structures. Figure 4 shows how the radial intensity profile evolves as transmitted $532 \mathrm{~nm}$ light continues to propagate along the $z$ direction away from the device plane. Intensity profiles obtained at $z=$ $0 \mu \mathrm{m}$ represent the optical images of the spiral zone structures. As the probed propagation distance is increased light becomes concentrated along the device axis. At distances of $f \approx 20 \mu \mathrm{m}(N A \approx 0.6)$ and $f \approx 15 \mu \mathrm{m}$ $(N A \approx 0.71)$ for the flat disk and obtuse axicon pedestals, respectively, intensity profiles converge into a well defined annular focus. The dark hollow at the center, as well as spiral order dependent radius of the spot are strongly indicative of an optical vortex. Overall the experimental results closely mirror behavior predicted by FDTD simulations, the only marked difference being the slightly longer measured focal distances $(f \approx 15 \mu \mathrm{m}$ instead of $f \approx 13 \mu \mathrm{m}$ for flat disk, and $f \approx 20 \mu \mathrm{m}$ instead of $f \approx 18 \mu \mathrm{m}$ for axicon pedestals), which can be attributed to phase front deviations due to a slight mismatch of the refractive indices of the polymer pedestal and the glass substrate. As the probing distance $z$ is increased further the beam starts to diverge, and at distances of $z=2 f$ is seen to reconstruct a flipped image of the spiral in the device plane.

Figure 5 shows closeup images of optical intensity at the focal planes as well as their interference patterns with a Gaussian reference arm. The experimentally measured fo- 
(a)
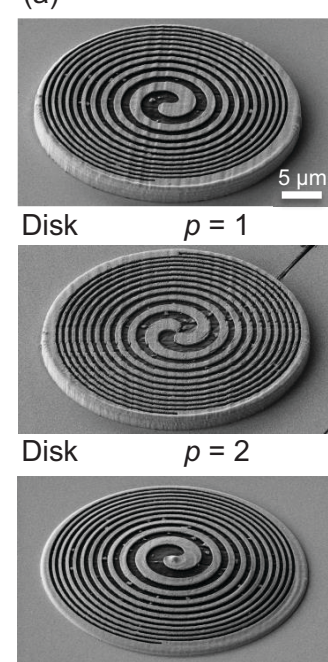

Axicon $p=1$

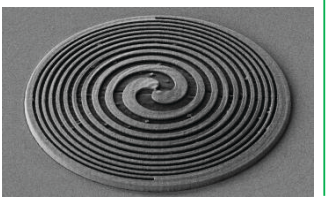

Axicon (b)
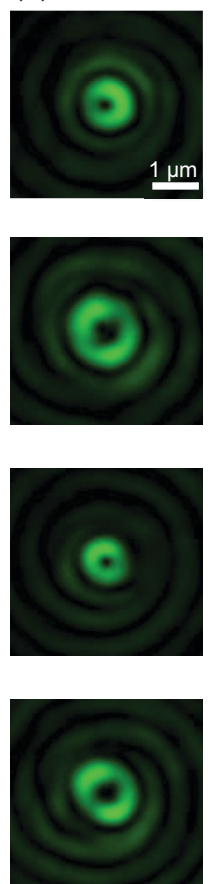

(c)
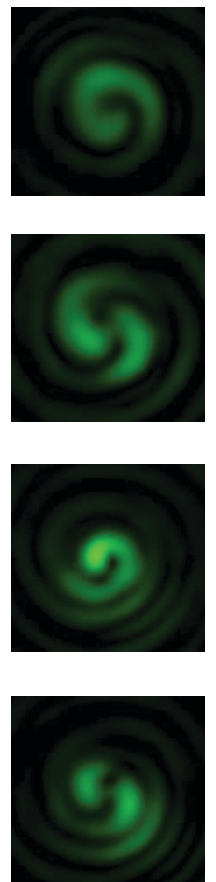

Figure 5. (b) Close up transverse cross-section of the vortex beam optical field at the focal plane of the SZP structures displayed in panel (a). (c) interference patterns at the focal plane with a collinear Gaussian reference arm, which reveal optical phase singularities with corresponding topological charges $\ell$. Imaging was done using a $100 x \mathrm{NA}=0.8$ objective.

cal spot diameters closely resemble their simulated counterparts, with $\sim 1.3 \mu \mathrm{m}$ and $\sim 2.0 \mu \mathrm{m}$ for vortices with topological charge of $\ell=1$ and $\ell=2$ respectively. The way axicon shaped substrates decrease the focal spot size is also visible from the imaging data. The topological charge states and any phase singularities present in the transmitted beam at the focal plane are unambiguously revealed by the interferograms presented in the column (c) of Fig. 5. Therein optical vortex with topological charge $\ell=1$ is observed for the first order spiral structures, and similarly $\ell=2$ is present for the second order spirals. This allows to conclude, based on both simulation and experimental results, that the spiral order directly corresponds to the topological charge $\ell$ of the highly focused vortex beam.

\section{Conclusions}

A novel 3D micro-optical element for highly focused vortex beam generation based on a binary spiral zone transmittance pattern on a photopolymerized pedestal was presented. Light propagation and focusing behavior was modeled using numerical FDTD simulations and predicted close to diffraction-limited focusing of a vortex beam at $f \approx 13-18 \mu \mathrm{m}$ distance from the device plane. Fabrication procedure for the proposed structures, based on combining the direct-write techniques of laser photopolymerization and FIB lithography, is outlined. Optical characterization of the fabricated structures revealed their propagation

properties and showed them to be in close accordance with theoretical predictions. This work shows how fabrication techniques combining refractive and diffractive optical approaches can allow for compound functionalities of optical elements at the microscale.

\section{Acknowledgements}

This work was partially supported by the Australian Research Council Discovery grant DP130101205 and the Linkage grant LP120100161 with Raith-Asia. DH and EB acknowledge support by the French State in the frame of the 'Investments for the future' Programme IdEx Bordeaux (reference ANR-10-IDEX-03-02). AZ and MM acknowledge funding by the Research Council of Lithuania under the Lithuanian-French Programme "Gilibert" (No. TAP LZ-4/2015). SJ acknowledges the startup funding of the Nanotechnology facility by strategic funding from Swinburne University. FDTD simulation work was performed on the swinSTAR supercomputer at Swinburne University of Technology.

\section{References}

[1] N.B. Simpson, D. McGloin, K. Dholakia, L. Allen, M.J. Padgett, J. Mod. Opt. 45, 1943-1949 (1998)

[2] M. Padgett, R. Bowman, Nature Photon. 5, 343-348 (2011)

[3] G.A. Swartzlander, Jr., Opt. Lett. 26, 497-499 (2001)

[4] G. Foo, D.M. Palacios, G.A. Swartzlander, Jr., Opt. Lett. 30, 3308-3310 (2005)

[5] J. Wang, J.Y. Yang, I.M. Fazal, N. Ahmed, Y. Yan, H. Huang, Y. Ren, Y. Yue, S. Dolinar, M. Tur et al., Nature Photon. 6, 488-496 (2012)

[6] L. Allen, M.W. Beijersbergen, R.J.C. Spreeuw, J.P. Woerdman, Phys. Rev. A 45, 8185-8189 (1992)

[7] G.F. Brand, Am. J. Phys. 67, 55 (1999)

[8] H. Liu, M.Q. Mehmood, K. Huang, L. Ke, H. Ye, P. Genevet, M. Zhang, A. Danner, S.P. Yeo, C.W. Qiu et al., Adv. Opt. Mater. 2, 1193-1198 (2014)

[9] Y. Gorodetski, A. Drezet, C. Genet, T.W. Ebbesen, Phys. Rev. Lett. 110, 203906 (2013)

[10] E. Brasselet, G. Gervinskas, G. Seniutinas, S. Juodkazis, Phys. Rev. Lett. 111, 193901 (2013)

[11] A. Žukauskas, M. Malinauskas, E. Brasselet, Appl. Phys. Lett. 103, 181122 (2013)

[12] N. Yu, P. Genevet, M.A. Kats, F. Aieta, J.P. Tetienne, F. Capasso, Z. Gaburro, Science 334, 333 (2011)

[13] L. Wei, Y. Gao, X. Wen, Z. Zhao, L. Cao, Y. Gu, J. Opt. Soc. Am. A 30, 233 (2013)

[14] A. Žukauskas, G. Batavičiūte, M. Ščiuka, T. Jukna, A. Melninkaitis, M. Malinauskas, Opt. Mater. Express 4, 1601 (2014)

[15] A. Ovsianikov, J. Viertl, B. Chichkov, M. Oubaha, B. MacCraith, I. Sakellari, A. Giakoumaki, D. Gray, M. Vamvakaki, M. Farsari et al., ACS Nano 2, 2257-2262 (2008) 